\title{
Preparation of alkali-resistant PVDF membranes via immobilization of sodium lauryl sulfate (SDS) on surface
}

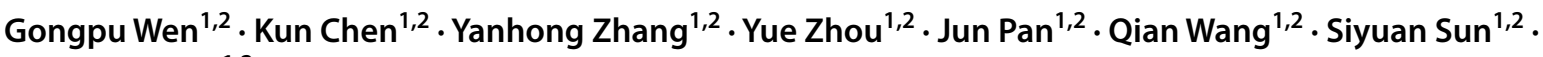 \\ Zhaohui Wang ${ }^{1,2}$
}

Received: 13 January 2021 / Accepted: 1 February 2021 / Published online: 13 March 2021

(c) The Author(s) 2021

\begin{abstract}
A novel strategy was proposed to fabricate alkali-resistant PVDF membrane via sodium lauryl sulfate (SDS) attached to the surface of membrane and immobilized by UV-curable polyester acrylate and tri(propylene glycol) diacrylate (TPGDA). The attached anionic surfactant, SDS, on the membrane surface can resist the alkali corrosion by $\mathrm{NaOH}$, and the curing of the resin can immobilize the SDS on the membrane firmly. Due to the unique alkali resistance of SDS and resin formed, the UV-curable resin-modified PVDF membrane showed greatly enhanced alkali-resistant ability. Characterization of SEM and FTIR showed that polyester acrylate and TPGDA were cured successfully under the action of 1-hydroxycyclohexyl phenyl ketone (184) and ultraviolet light. Whiteness, differential scanning calorimeter and X-ray photoelectron spectrometer characterization showed that the modified PVDF membrane had a lower degree of dehydrofluorination than the pristine PVDF membrane after alkali treatment. Results of the detailed alkali-resistant analysis indicated that the F/C ratio of the UV-curable resin-modified PVDF membrane decreased by $2.6 \%$ after alkali treatment compared to pristine PVDF membrane decreased by $19.28 \%$. The alkali-resistant performance was mainly attributed to the immobilized SDS. This study provided a facile and scalable method for designing alkali-resistant PVDF membrane, which shows a promising potential in the treatment of alkaline wastewater and alkaline-cleaning PVDF membrane.
\end{abstract}

Keywords Poly(vinylidene fluoride) membrane $\cdot$ Alkali resistance $\cdot$ UV curing

\section{Introduction}

Poly (vinylidene fluoride) (PVDF) membrane has excellent chemical and physical resistance, strong mechanical strength and good thermal stability (Yeow et al. 2003; Hashim et al. 2009; Liu 2011), in which these properties make it suitable for wastewater treatment. Although PVDF has excellent chemical stability to various harsh chemicals (such as inorganic acids, oxidants and halogens), it is not sufficient to the strong alkali solution $(\mathrm{pH} \geq 12)$ (Rabuni 2013). High alkali resistance is very important because alkali cleaner can effectively remove irreversible foulants to recover the membrane

Zhaohui Wang

zhwang@njtech.edu.cn

1 State Key Laboratory of Materials-Oriented Chemical Engineering, College of Chemical Engineering, Nanjing Tech University, Nanjing 210009, China

2 National Engineering Research Center for Special Separation Membrane, Nanjing Tech University, Nanjing 210009, China flux and $\mathrm{NaOH}$ solution $(0.2 \mathrm{M})$ was usually used as liquid absorbents in the membrane contactor (Mansourizadeh et al. 2010). For example, rinsing the membrane fouled by humic acid with $0.1 \mathrm{~mol} / \mathrm{L} \mathrm{NaOH}$ solution can get $100 \%$ flux recovery (Srisurichan et al. 2005). Water/NaOH (1 wt \%) was employed for alkaline cleaning of heavily contaminated PVDF membranes in a submerged rotating membrane bioreactor system (Zuo 2010). After cleaning, obvious membrane pores appeared, while the $\mathrm{NaOH}$ solution can effectively remove the organic foulants of proteins (Yu 2013; Li 2008; Wang 2014). $\mathrm{NaOH}$ solution ( $0.5 \mathrm{M}$ ) was used to effectively remove $\mathrm{CO}_{2}$ from natural gas in the PVDF membrane contactor (Ghasem et al. 2012).

However, frequent alkali cleaning and long-term $\mathrm{NaOH}$ solution that is used for PVDF membrane system will reduce its mechanical properties and shorten its life. Therefore, PVDF is a membrane that cannot be frequently chemically cleaned for a long time and used to handle alkaline wastewater. The application range of PVDF membrane materials is subject to certain restrictions. Many reports 
have fundamentally confirmed that PVDF membrane materials were susceptible to damage by alkaline conditions (Komaki and Otsu 1981; Shinohara 1979; Kise and Ogata 1983; Wegener et al. 2006; Vigo 1984). It was observed that the color of the PVDF membrane changed from white to brown and then to black after exposure in the $\mathrm{NaOH}$ solution (Komaki and Otsu 1981; Shinohara 1979); thus, it becomes brittle (Komaki and Otsu 1981; Shinohara 1979; Antón 2015; Ross 2000). This is interpreted as a dehydrofluorination reaction, which removes hydrogen fluoride (HF) units from the polymer chain to form a $\mathrm{C}=\mathrm{C}$ bond (Kise and Ogata 1983; Ross 2000; Zhang 2006; Kuhn 1987). Hashim et al. found that the reaction between PVDF and $\mathrm{NaOH}$ was triggered even at low concentrations of $\mathrm{NaOH}$ and was aggravated with the extended treatment time, resulting in the decrease in mechanical strength and crystallinity of PVDF. The reaction was accelerated and intensified by increasing the concentration of $\mathrm{NaOH}$ and treatment temperature. Rabuni et al. confirmed that PVDF membrane is more vulnerable to $\mathrm{NaOH}$ solution as compared to $\mathrm{KOH}$ solution (Rabuni 2013; Hashim et al. 2011a; Jang 2017; Wu et al. 2018). Such characterization and conclusion provide basic information on the stability of PVDF membrane materials in harsh caustic environments. However, these investigations only explored the information of the degradation process of PVDF in alkaline environments, and there was little research on the alkali-resistant modification of PVDF membranes, which led to the fact that the PVDF membranes cannot be chemically cleaned by $\mathrm{NaOH}$ and treat alkali wastewater.

In this article, the research of PVDF membrane alkali resistance modification was reported. PVDF membranes with appropriate pore size prepared by non-solvent-induced phase separation (NIPS) were pre-modified by coating gelatin to design a universal "biological glue" layer, which fixed sodium lauryl sulfate (SDS) initially that played a vital role in resisting the nucleophilic attack of the $\mathrm{NaOH}$ solution. But SDS is a water-soluble substance, along with the increase of the running time of the pre-modified membrane in the process of testing pure water flux; SDS wrapped in gelatin was washed away. Its ability to resist alkali corrosion declined rapidly. Due to the lack of practicality, the pre-modified PVDF membrane was selectively characterized in this paper. In order to solve this problem, resin was used to encapsulate SDS in "biological glue" layer which pre-modified on the PVDF membrane. The cured resin layer formed by the polyester acrylate and tri(propylene glycol) diacrylate (TPGDA) was firmly immobilized the "biological glue" layer on the PVDF membrane. The excellent alkali resistance and waterproof performance of the resin formed (Cao and Zheng 2005) could effectively prevent the loss of SDS during the operation of membrane to achieve the performance of long-term alkali resistance of the PVDF membrane. The characterization results showed that the degree of dehydrofluorination of the UV-curable resin-modified PVDF membrane was much smaller than that of the pristine membrane under the same alkaline conditions.

\section{Experimental}

\section{Material}

Commercially available PVDF (Solef ${ }^{\circledR}$ 6010) was obtained from Solvay Specialty Polymers. Dimethylacetamide (DMAc) (> 99.8\%), tri(propylene glycol) diacrylate (TPGDA) (>99.99\%), 1-hydroxycyclohexyl phenyl ketone (184) and polyvinylpyrrolidone (PVP K30) (Mw: 44,000-54,000) were purchased from Shanghai Aladdin Reagent Co., Ltd. Hydrophilic nano-SiO 2 (BET: $380 \mathrm{~m}^{2} / \mathrm{g}$, particle size: $7-40 \mathrm{~nm}$ ), sodium dodecyl sulfate (SDS) (>99.5\%), gelatin (Mn: 50,000-100,000) and dichloromethane $(\geq 99.9 \%)$ were obtained from Shanghai Macklin Biochemical Co., Ltd. Polyester acrylate (Mn: 1011, Mw: 1259) was provided by Guangdong Ketian Chemical Materials Co., Ltd.

\section{Fabrication of PVDF/nano-SiO ${ }_{2}$-blended membrane}

The membrane was prepared by blending $15 \mathrm{~g}$ PVDF (Solef® 6010) and $2.0 \mathrm{~g}$ hydrophilic nano- $\mathrm{SiO}_{2}$ in $60 \mathrm{~g}$ DMAc. Two grams of PVP K30 was also added to finely tune the porosity and pore size of the membrane. The mixed solution was kept stirring for $12 \mathrm{~h}$. After stirring, degassing of the solution was done by keeping the solution stable at $60{ }^{\circ} \mathrm{C}$ for $24 \mathrm{~h}$. Afterward, the solution was poured on a clean glass-plate and spread using a casting knife (the height of the knife was adjusted to $250 \mu \mathrm{m}$ and the speed was adjusted to $1.2 \mathrm{~m} / \mathrm{min}$ ). Then the glass-plate with nascent membrane was immersed into coagulation bath containing $15 \%$ DMAc aqueous solution at $60{ }^{\circ} \mathrm{C}$. After the coagulation process was completed, the membrane was transferred into $4.0 \%$ sodium hypochlorite solution and kept overnight to remove the additional solvent and PVP K30 from the membrane.

\section{Pre-modified membrane by immersion coating}

Five grams of gelatin and $10 \mathrm{~g}$ of sodium lauryl sulfate were dissolved in $90 \mathrm{~g}$ DI water under mechanical stirring at $40{ }^{\circ} \mathrm{C}$, and the PVDF membrane prepared above was immersed in this solution for six hours. The PVDF membrane was then removed from the solution and dried naturally. 


\section{Immobilization of the pre-modified PVDF membrane by UV-curable resin}

$1.5 \mathrm{~g}$ of polyester acrylate, $0.2 \mathrm{~g}$ of 1-hydroxycyclohexylphenylketone (184) and $5.0 \mathrm{~g}$ of dichloromethane were dissolved in $12 \mathrm{~g}$ TPGDA under mechanical stirring at $40{ }^{\circ} \mathrm{C}$ hermetically. After the prepared polyester acrylate resin solution cooled completely, the pre-modified PVDF membrane was immersed in the resin solution configured above for $24 \mathrm{~h}$. It was observed that the PVDF membrane was completely wetted with the polyester acrylate resin solution, the wetting state of PVDF membrane by polyester acrylate resin solution was similar to the wetting state of PVDF membrane by alcohol. The wetted PVDF membrane was put forward from the polyester acrylate resin solution, and the excess resin on the surface of the PVDF membrane was wiped by a nonwoven fabric. Then, the membrane was placed under a 1000-w UV lamp for radiation curing. The vertical distance between the UV lamp and membrane was $35 \mathrm{~cm}$. Different UV curing times ( $30 \mathrm{~s}, 40 \mathrm{~s}, 50 \mathrm{~s}, 60 \mathrm{~s}, 70 \mathrm{~s}, 80 \mathrm{~s}, 90 \mathrm{~s}$ ) were investigated. Subsequently, the membrane was immersed in ethanol solution for $6 \mathrm{~h}$ to remove the unreacted TPGDA and polyester acrylate, and the membrane was transferred to DI water for subsequent experiments and characterization.

\section{Material and membrane characterization techniques}

The morphology of the surface and cross section of the PVDF membranes were inspected by the cold field emission scanning electron microscope (FESEM; S-4800, HITACHI, Tokyo, Japan). The dried membrane samples were placed on the conductive adhesive of the metal bracket and then sputter-coated using gold under vacuum for $40 \mathrm{~s}$. The images of the surface of the pristine PVDF membrane (M-Pristine), UV-curable resin-modified PVDF membrane (M-UV curing), alkali-treated M-Pristine and alkali-treated M-UV curing were observed at a range of magnifications. The dried membrane samples were immersed in liquid nitrogen for about 5-10 min. Subsequently, the samples were broken with tweezers in order to obtain cross-sectional fracture of the membranes.

Edborg digital display push-pull force gauge HP-30N (Edborg, China) was used to measure the membrane's mechanical strength. Each sample was cut into the same shape with a Japanese knife mold, and the thickness of each sample was measured using an electronic digital membrane thickness gauge before testing. Both ends of the samples were fixed and stretched at a constant rate of $5 \mathrm{~mm} / \mathrm{min}$ $\left(25{ }^{\circ} \mathrm{C}\right)$. Each sample was measured for five times and the average data from the remaining measurements.

X-ray diffractometer (XRD, RIGAKU MiniFlex600, Japan) was used to measure crystalline structure of PVDF membrane.
Sample was positioned on a sample holder loaded on the diffractometer. The scanning range covered $0^{\circ}<2 \theta<60^{\circ}$ with a scanning step of $0.02^{\circ}$.

Fourier transform infrared (FTIR) spectrometer (Thermo Nicolet8700, USA) equipped with an attenuated total reflection (ATR) accessory was used to analyze the changes of M-Pristine, M-UV curing, alkali-treated M-Pristine and alkali-treated M-UV curing in chemical functional groups. The samples were soaked twice with ethanol and then washed twice with water to remove impurities and solvents and dried. The pretreated samples were positioned on the sample holder, and the spectra in the wavenumber range of $4000-400 \mathrm{~cm}^{-1}$ were recorded by accumulating 64 scans with a resolution of $0.4 \mathrm{~cm}^{-1}$.

Whiteness tester (WSB-2, China) was used to measure the whiteness of the sample. Black tube was placed on the sample holder at the bottom of the instrument. When the value on the display was stable, the adjustment knob was turned to make the value on the display be 00.0 . Then, the black tube was removed and put on the working standard whiteboard. When the value on the display was stable, the correction knob was adjusted on the panel to make the displayed value equal to the whiteness value of the working standard whiteboard. The working standard whiteboard was removed, and the reference standard whiteboard was placed on the sample holder at the bottom of the instrument. When the value on the display was stable, the whiteness value was record as the standard value of the sample.

$\mathrm{X}$-ray photoelectron spectrometer (ESCALAB 250Xi, USA) was used to determine the type and relative content of elements on the sample surface. For the accuracy of elemental quantitative analysis, samples were prepared in a vacuum glove box to ensure that the sample surface was clean.

A differential scanning calorimeter (DSC TA Q-20, UK) was used to obtained thermograms at a heating rate of $10^{\circ} \mathrm{C} /$ min for all the specimens.

Contact angle tester (Dropmeter A-100, China) was used to test the hydrophilicity and wettability of the samples. A pore size distribution instrument (MCE-PDA-10, China) was used to test the pore size distribution of the samples. A selfassembled pure water flux testing instrument (self-assembly in the laboratory) was used to obtain pure water flux of samples (Scheme 1).

To ensure the continuity and stability of the nucleophilic substitution reaction of $\mathrm{NaOH}$ and PVDF, the sodium hydroxide aqueous solution was renewed once per day. 
Scheme 1 Schematic diagram of pure water flux measurement

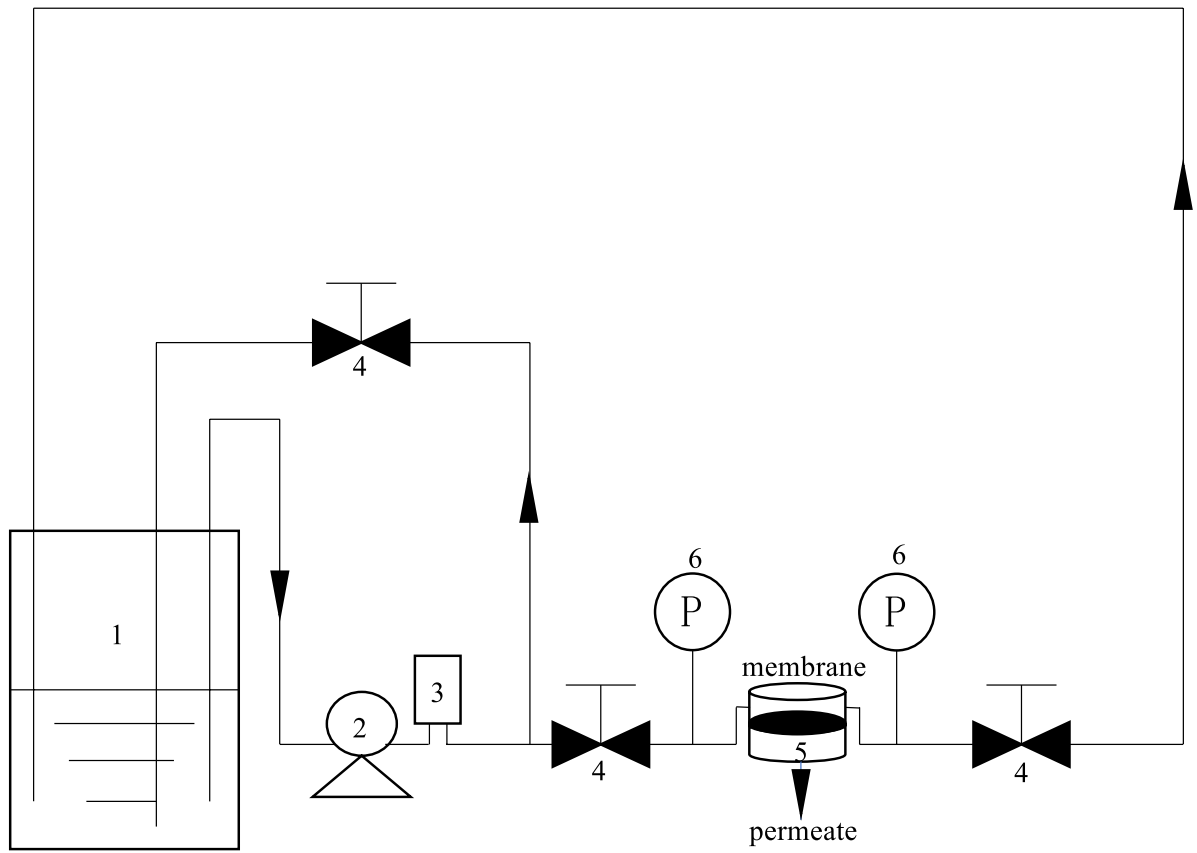

1.DI water tank 2.pump 3.buffer 4.valve 5.Membrane module 6.Pressure gauge

\section{Results and discussion}

\section{Whiteness}

According to the previous investigations, the corrosion degree of PVDF membrane by alkaline solution can be directly observed by PVDF membrane color (Hoa and Ouellette 1983; Kise et al. 1989; Liu 2017; Rabuni 2013), so the whiteness characterization was used to record the color change of the PVDF membrane by NAOH $(\mathrm{pH}=13)$ corrosion. Figure 1 shows the pictures of the pristine PVDF membrane and the UV-curable resin-modified PVDF membrane before and after immersion in $\mathrm{NaOH}$

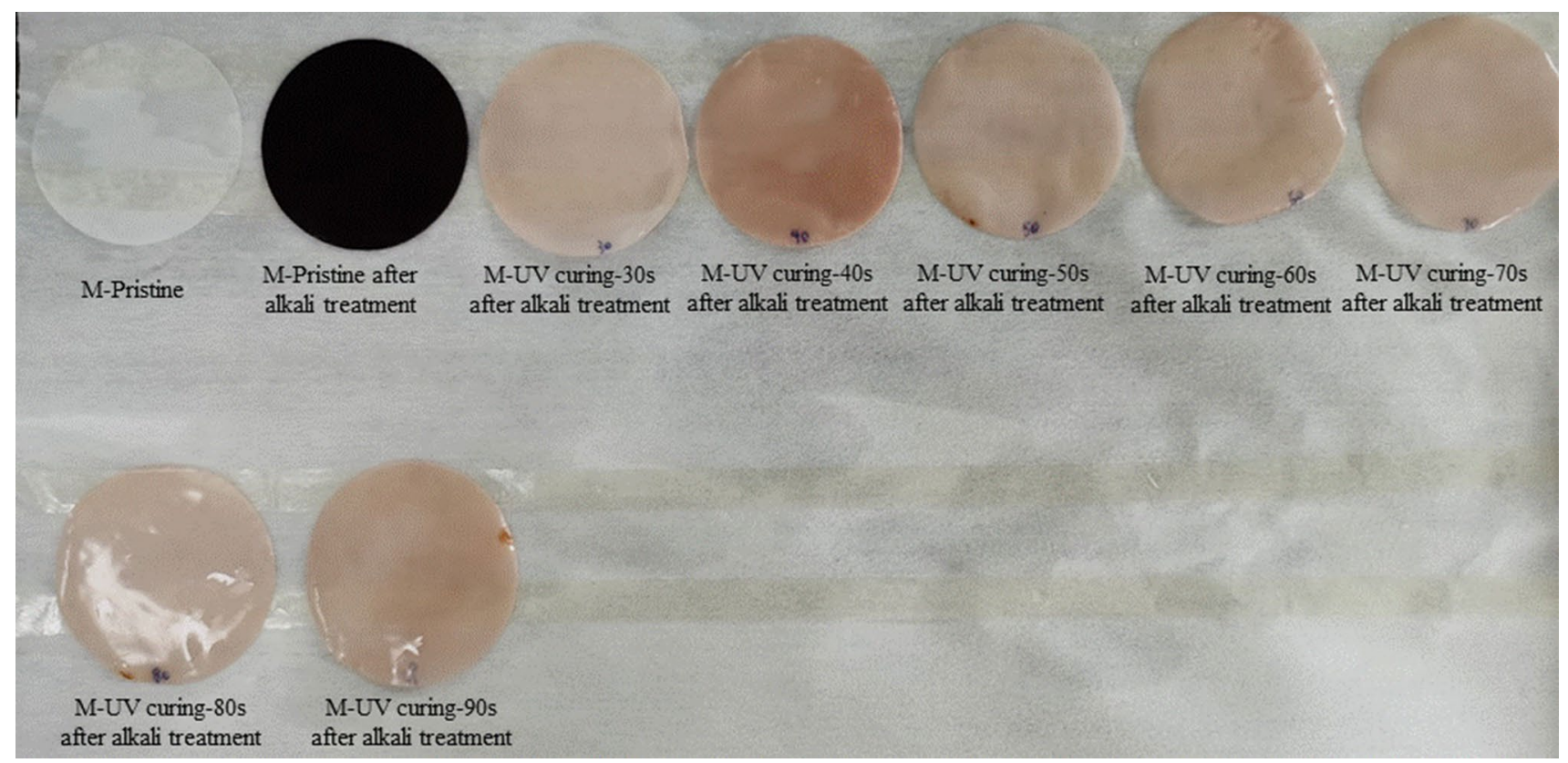

Fig. 1 Effect of $\mathrm{NaOH}$ treatment on whiteness 
aqueous solution $(\mathrm{pH}=13)$. When the pristine PVDF membrane was immersed in the alkaline solution, the surface color changed from white to brown and finally to black after one month, which has been reported in other

Table 1 The effect of $\mathrm{NaOH}$ treatment on whiteness

\begin{tabular}{ll}
\hline Specimen & Whiteness \\
\hline Reference standard whiteboard & 79.7 \\
Black tube & 0 \\
M-Pristine & 29.7 \\
M-UV curing & 29.7 \\
M-Pristine after alkali treatment (30 days) & 1.6 \\
M-UV curing-30 s after alkali treatment (30 days) & 21.1 \\
M-UV curing-40 s after alkali treatment (30 days) & 18.2 \\
M-UV curing-50 s after alkali treatment (30 days) & 19.8 \\
M-UV curing-60 s after alkali treatment (30 days) & 21.1 \\
M-UV curing-70 s after alkali treatment (30 days) & 19.9 \\
M-UV curing-80 s after alkali treatment (30 days) & 21.7 \\
M-UV curing-90 s after alkali treatment (30 days) & 19.4 \\
\hline
\end{tabular}

studies (Komaki and Otsu 1981; Shinohara 1979). It can contribute to the fact that the $\mathrm{C}=\mathrm{C}$ bond formed by dehydrofluorination in the polymer chain causes color change in PVDF. However, the color of the UV-curable resinmodified PVDF membrane has just changed to slightly brown.

It can be observed from Table 1 that the whiteness of the pristine PVDF membrane decreased greatly by $94.61 \%$ after the alkali treatment in thirty days, which was almost close to the black tube, due to the severe dehydrofluorination. On the contrary, the whiteness of the UV-curable resin-modified PVDF membrane decreased slightly by between 26.94 and $38.72 \%$ after alkali treatment within one month, indicating that the alkali-resistant layer effectively shielded the nucleophilic attack of $\mathrm{NaOH}$ on PVDF.

\section{Morphology of PVDF membranes}

The effect of alkali solution on the surface morphology of PVDF membranes was further investigated by FESEM analysis. Figure 2 shows the SEM images of the PVDF
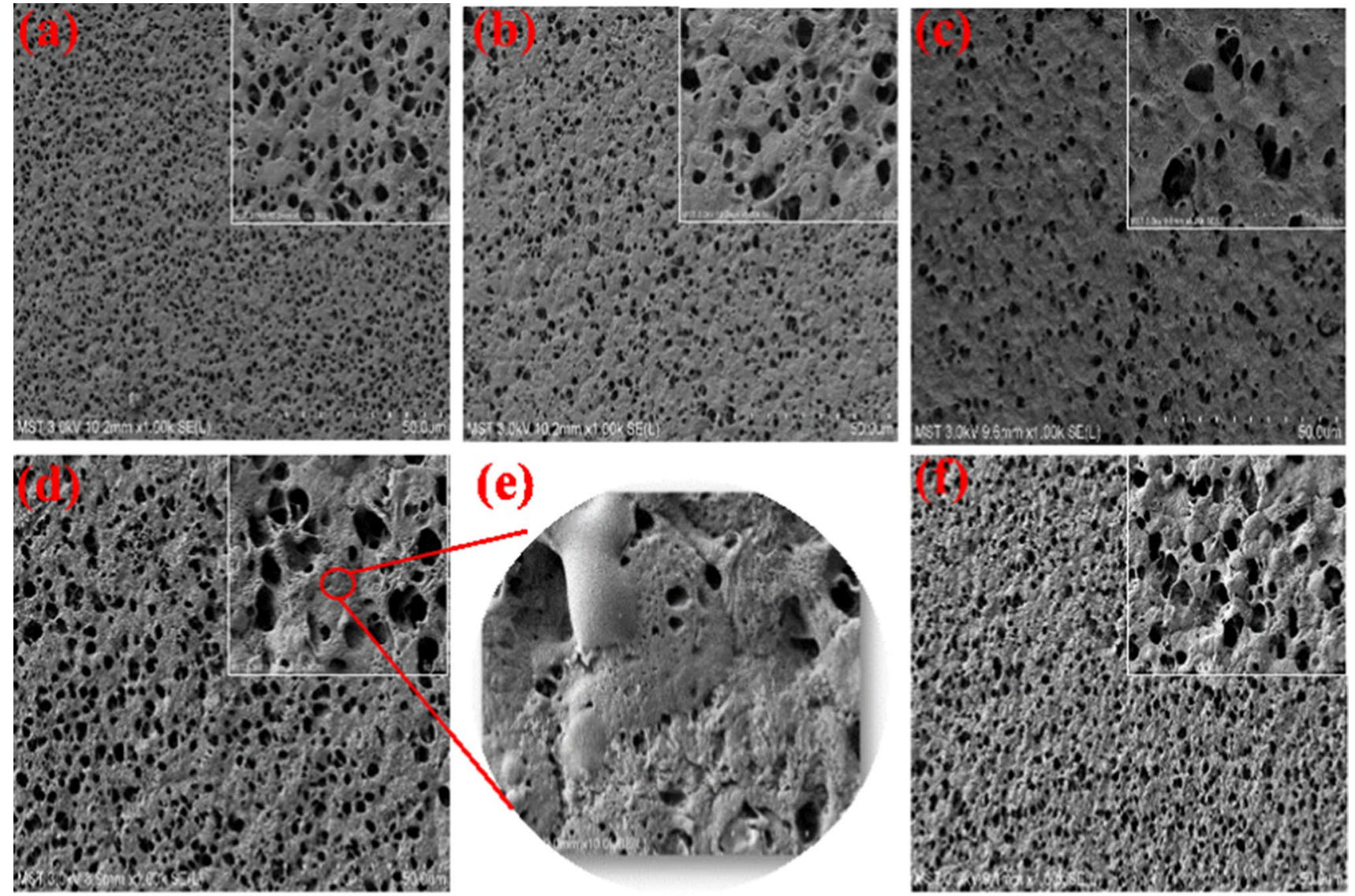

Fig. 2 Surface images of a M-Pristine, b M-coating, c M-Pristine after alkali treatment $(\mathrm{NaOH}$ (30 days), $\mathrm{pH}=13)$, d, e M-UV curing, f M-UV curing after alkali treatment $(\mathrm{NaOH}$ (30 days), $\mathrm{pH}=13)$. All images shown are the respective surfaces in contact with the glass substrate during membrane casting by phase inversion 
membrane surface before and after immersion in alkaline solution. In order to better explain the change of membrane surface morphology after resin modification, the glass side of membrane was used as the main analysis surface of the PVDF membrane. From Fig. 2a, it can be observed that the surface of the pristine PVDF membrane consists of uniformly distributed pores. As shown in Fig. 2b, the surface of the membrane still retained its pristine apparent morphology after being pre-modified by immersion coating. For the pristine membrane treated with alkaline solution, as shown in Fig. 2c, the surface of the membrane did not change significantly in general compared with the pristine membrane. Hashim also observed the same phenomenon that there were no significant changes in the PVDF membrane surface after $\mathrm{NaOH}$ treatment (Hashim et al. 2011a). After modification of the UV-curable resin, it can be intuitively observed that the resin formed by polyester acrylate and TPGDA perfectly fixed on the surface of the membrane, and the phenomenon of large-area plugging did not occur. More details can be seen in Fig. 2d. In order to avoid the decrease of membrane flux, dichloromethane, a low-boiling, highly volatile solvent, was added to the resin solution. During the UV-curing process, the temperature gradually increased, and dichloromethane volatilized and caused pores, as shown in Fig. 2e at 10,000 magnifications. After the alkaline treatment, as shown in Fig. 2f, the surface morphology of UV-curable resin-modified PVDF membrane has not changed significantly compared to Fig. 2d, e.
The cross-section SEM images of PVDF membranes are provided in Fig. 3. It can be seen from Fig. 3a that the cross section of the pristine PVDF membrane is a sandwich structure with a thin skin layer at upper side, finger-like structure at middle, and the lower layer is a thick sponge-like structure. It can be seen from Fig. $3 b$ that the cross-section structure of pre-modified PVDF membrane is not much different from the pristine PVDF membrane.

Compared with Fig. $3 a$, b, it is obvious that the cross section of the PVDF membrane changed significantly from Fig. $3 c$ under the same SEM detection conditions, the resin formed by polyester acrylate and TPGDA firmly encapsulated the cross-section structure of the PVDF membrane. The UV-curable resin-modified PVDF membrane largely maintained the cross-sectional structure of the pristine PVDF membrane without blocking the finger-like channels of PVDF membrane by the resin during the UV-curing process.

After treated with alkaline solution, as shown in Fig. 3d, the cross-sectional morphology of UV-curable resin-modified PVDF membrane has not changed significantly compared to Fig. 3c.

\section{Mechanical strength}

The mechanical stability of the PVDF membrane was evaluated by measuring the tensile strength. Mechanical stability measurement is very important, because the use life of the membrane can be estimated by examining the mechanical
Fig. 3 Cross-section images of a M-Pristine, b M-coating, c M-UV curing, $\mathbf{d}$ M-UV curing after alkali treatment
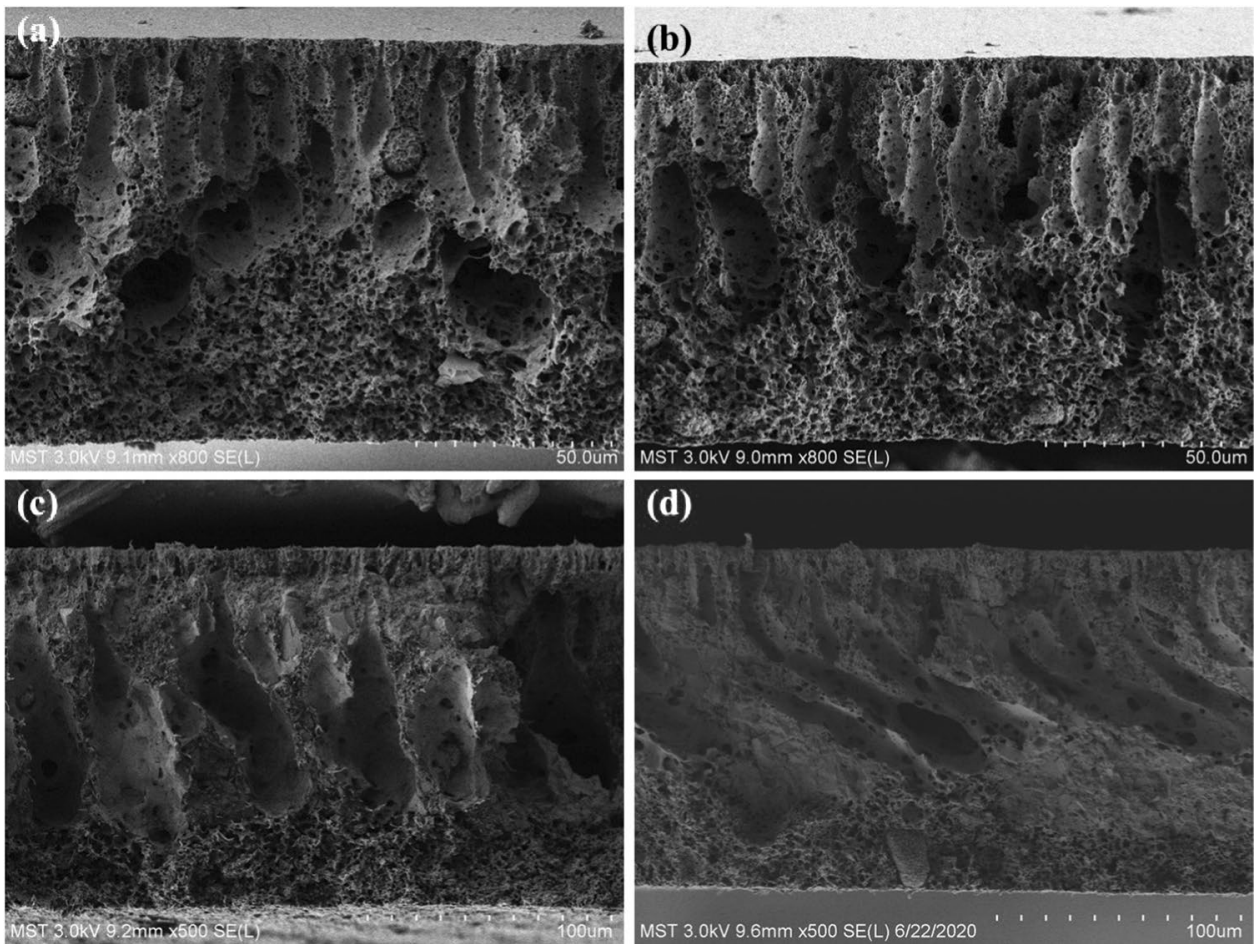
(a)

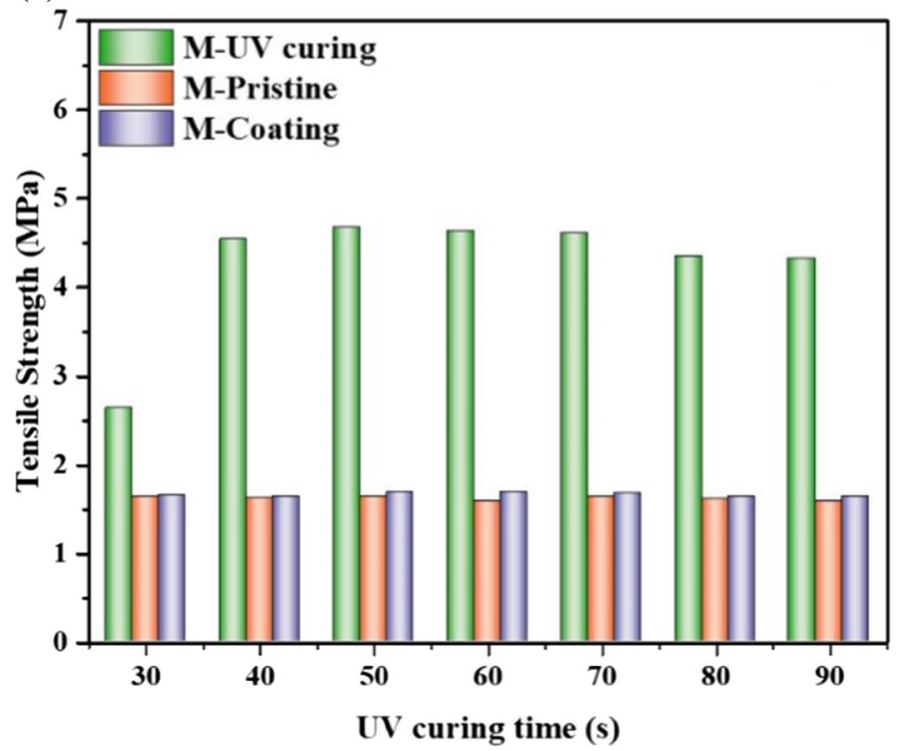

(b)

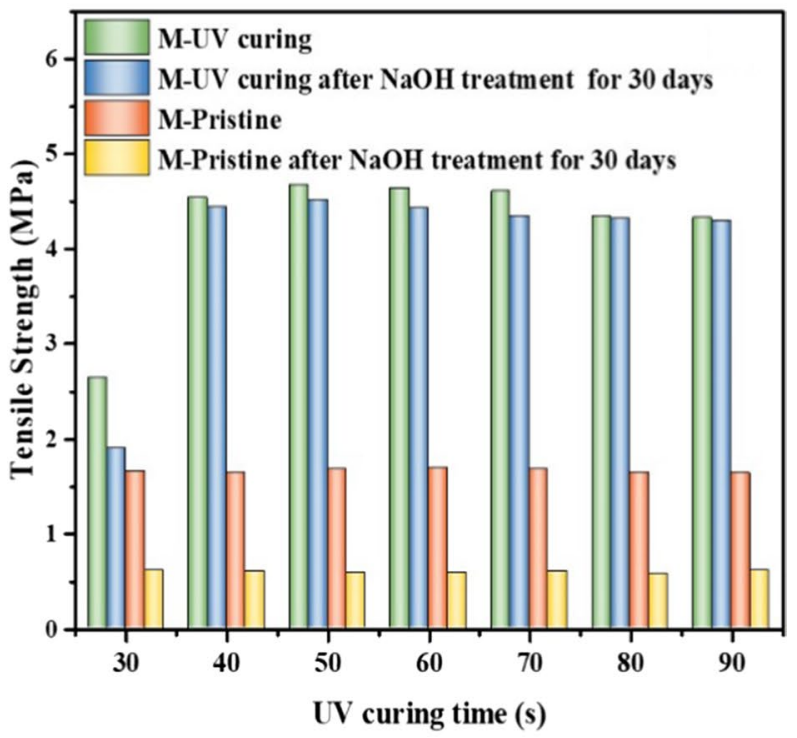

Fig. 4 a Effect of UV-curable resin-modified PVDF membrane on tensile strength (there is no relationship between the UV curing time of abscissa and M-Pristine and M-coating). b Effect of $\mathrm{NaOH}$ $(\mathrm{pH}=13)$ treatment on pristine PVDF membrane and UV resin cured modified PVDF membrane at room temperature (there is no relationship between the UV curing time of abscissa and M-Pristine, as well as M-Pristine after $\mathrm{NaOH}$ treatment)

which indicates that the influence of $\mathrm{NaOH}$ on the UV-curable resin-modified PVDF membrane is much lower than that of the pristine PVDF membrane. The well mechanical properties of UV-curable resin-modified PVDF membranes after alkali treatment for one month showed that the degradation velocity of UV-curable resin-modified PVDF membrane was much lower than pristine PVDF membrane. The resin layer perfectly encapsulated the pristine PVDF membrane and the sodium lauryl sulfate present in the coating layer. It effectively shielded the PVDF from the chemical attack of the strong nucleophilic $\mathrm{NaOH}$ aqueous solution, thereby significantly improving the alkali resistance of the PVDF membrane.

\section{XRD analysis}

PVDF is a semi-crystalline polymer and it has at least four phases: $\alpha, \beta, \gamma$ and $\delta$. Different phases perform differently in filtration and anti-fouling process (Lovinger 1983). So it is important to investigate the effect of alkali on the PVDF polymorphsim. XRD analyses were performed to examine the crystal structures and structural changes of PVDF after membrane formation. It can be deduced that $\alpha$-phase dominated in the pristine PVDF membrane and UV-curable resin-modified PVDF membrane from the XRD pattern, for $18.30^{\circ}, 19.9^{\circ}$ and $26.56^{\circ}$, were the characteristic peaks for $\alpha$-phase PVDF (Wu et al. 2018). In addition, the crystal structure of the membrane did not change after the UVcurable resin modification (Fig. 5). slightly decreased after soaking in $\mathrm{NaOH}$ for one month, 


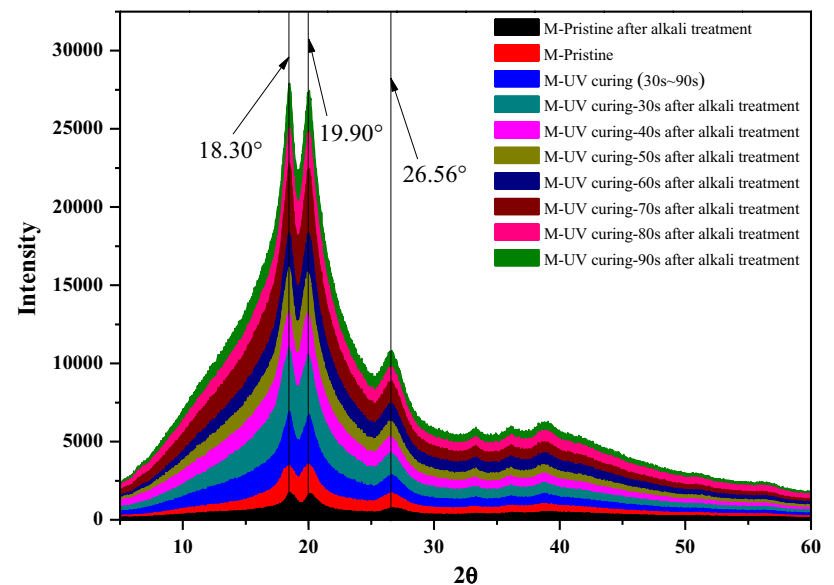

Fig. 5 The effect of alkali treatment on the crystal form of pristine PVDF membrane and UV-curable resin-modified PVDF membrane (the XRD spectrum of the UV-curable resin-modified PVDF membrane (30-90 s) is basically same)

On the other hand, no significant changes were detected by the XRD analysis on the crystal structure of pristine PVDF membranes after alkali treatment as well as UV-curable resin-modified PVDF membrane indicating that alkali treatment did not change the crystal structure of the membrane. Hashim et al. (2011a) and Wu et al. (2018) also have found the same phenomenon and drawn the same conclusion that alkali treatment may not change the crystal structure of the PVDF membrane.

\section{ATR-FTIR spectroscopy}

In this study, ATR-FTIR analyses were conducted to determine the crystal structure and changes of chemical functional groups from the pristine PVDF membrane and the UV-curable resin-modified PVDF membrane before and after alkali treatment.

ATR-FTIR spectra of pristine PVDF membrane and UV-curable resin-modified PVDF membrane are illustrated in Fig. 6a, b. As seen from Fig. 6a, characteristic bands appeared at 762, 796, 876, 1070, 1178 and $1423 \mathrm{~cm}^{-1}$, which were similar to the bands observed for PVDF membrane containing mainly $\alpha$-phase, while several other peaks observed at 840 and $1402 \mathrm{~cm}^{-1}$ could be attributed to $\beta$-phase (Cui 2015; Boccaccio 2002).

As illustrated in Fig. 6b, the infrared characteristic absorption peaks appearing at 741 and $1730 \mathrm{~cm}^{-1}$, respectively, were considered to be $\mathrm{C}-\mathrm{Cl}$ and $\mathrm{C}=\mathrm{O}$ characteristic bonds, since there are a large number of $\mathrm{C}=\mathrm{C}, \mathrm{C}=\mathrm{O}$ and $\mathrm{C}-\mathrm{Cl}$ groups in the polyester acrylate and $\mathrm{C}=\mathrm{C}$ and $\mathrm{C}=\mathrm{O}$
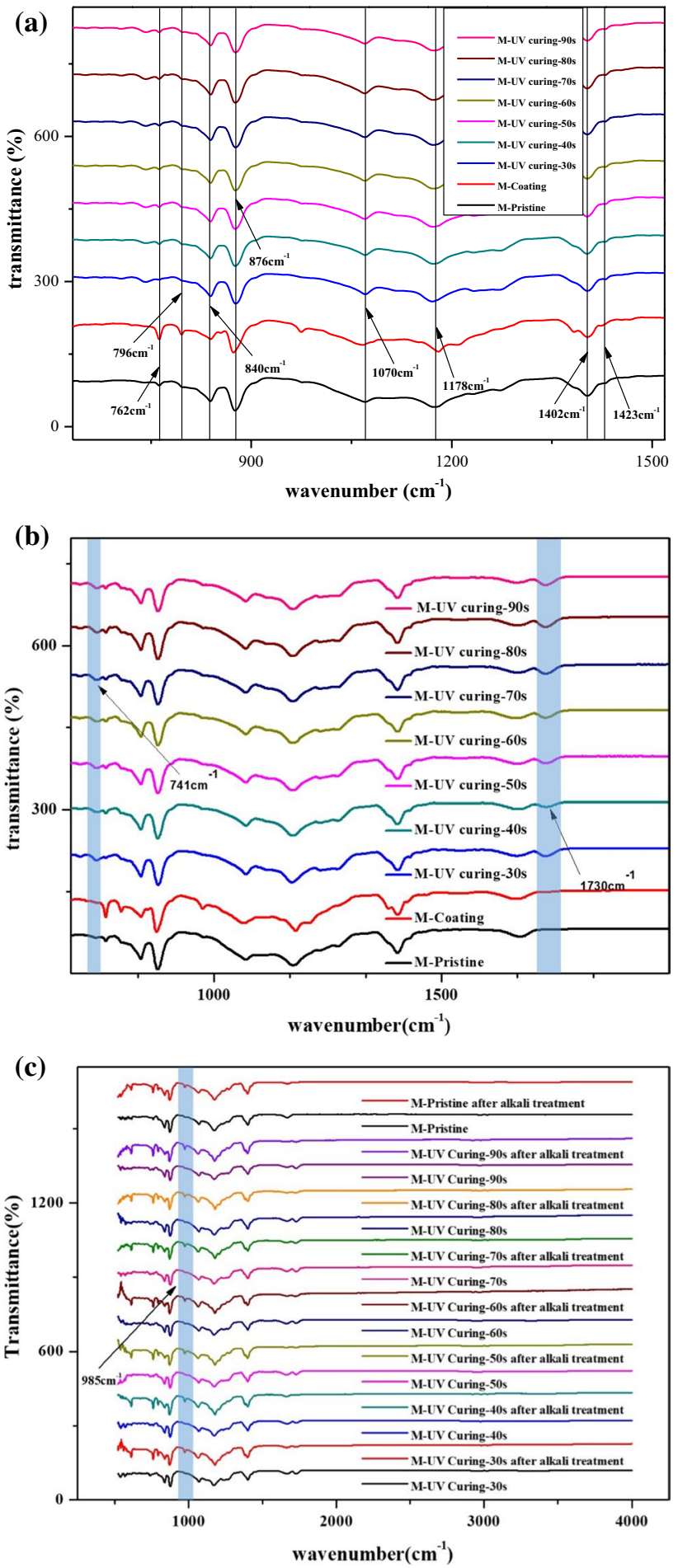

Fig. 6 a Changes of crystal form in ATR-FTIR spectrum after UVcuring resin modification. b Changes of functional groups in ATRFTIR spectrum after UV-curing resin modification. c Changes of functional groups of pristine PVDF membrane and UV-curable resinmodified PVDF membrane after alkali treatment 
groups in the TPGDA. Under the action of ultraviolet light and photoinitiator 184 , the polyester acrylate $\mathrm{C}=\mathrm{C}$ bond was functioned as the reaction site for free radical copolymerization with the $\mathrm{C}=\mathrm{C}$ bond in TPGDA. After the ultraviolet curing, the mixed oily solution of polyester acrylate and TPGDA attached to the pre-modified PVDF membrane became solid polymer. As mentioned in Fig. $6 \mathrm{~b}$, the infrared absorption peaks of the $\mathrm{C}-\mathrm{Cl}$ and $\mathrm{C}=\mathrm{O}$ bonds were detected in the infrared spectrum, which proved that the polyester acrylate and TPGDA were successfully cured. (The resin formed was also detected on the surface and section of the membrane in SEM shown in Figs. 2d, e, 3c.)

The changes in the functional groups of pristine PVDF membrane and UV-curable resin-modified PVDF membrane caused by the $\mathrm{NaOH}$ treatment at room temperature $\left(25^{\circ} \mathrm{C}\right)$ were studied by ATR-FTIR. The dehydrofluorination effect was caused by $\mathrm{NaOH}$ treatment forming the $\mathrm{C}=\mathrm{C}$ bond, which has been proved by previous investigation (Hoa and Ouellette 1983; Kise et al. 1989). Due to the addition of the hydrophilic additive PVP K30, the infrared absorption peak of the $\mathrm{C}=\mathrm{O}$ group of PVP $\mathrm{K} 30$ and the infrared absorption peak of the $\mathrm{C}=\mathrm{C}$ bond coincide with each other, resulting in invisibility in ATR-FTIR. However, the $\mathrm{C}-\mathrm{H}$ group in $(-\mathrm{CF}=\mathrm{CH}-)$ generated by dehydrofluorination showed a peak phenomenon between 970 and $990 \mathrm{~cm}^{-1}$ in ATRFTIR spectra. At the wave number of $985 \mathrm{~cm}^{-1}$, no peak was detected in the pristine membrane and the UV-curable resin-modified PVDF membrane that was not treated with the $\mathrm{NaOH}$ aqueous solution, while appeared after the $\mathrm{NaOH}$ treatment. Even in the UV-cured resin-modified membrane that was slightly corroded by alkali, a peak at $985 \mathrm{~cm}^{-1}$ in FTIR spectra was also detected. We believe that even a slight dehydrofluorination phenomenon caused by alkali treatment will have an infrared absorption peak with a clear wavenumber in the infrared.

\section{Thermal analysis using DSC}

The effect of alkaline treatment toward membrane's thermal properties was investigated based on the melting temperature and enthalpy from DSC analysis. There was almost no change DSC spectrum on the UV-curable resinmodified PVDF membrane (30-90 s) treated by alkali solution. Therefore, the PVDF membrane with a curing time of $40 \mathrm{~s}$ was chosen to represent all the alkali-treated UV-cured membranes.

As shown in Fig. 7, the melting temperature and melting enthalpy of the pristine PVDF membrane were the same as the UV-curable resin-modified membrane. The resin wrapping PVDF membrane has a melting temperature of $307.11^{\circ} \mathrm{C}$. When the temperature rose to about $170{ }^{\circ} \mathrm{C}$, the PVDF membrane wrapped in the resin absorbed heat and melted. According to previous literature (Kise and

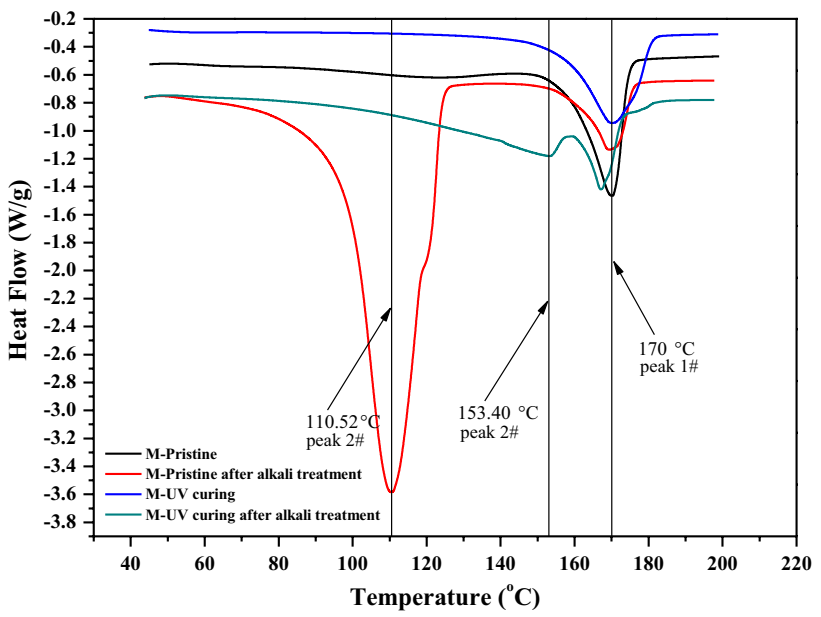

Fig. 7 DSC curves of $\mathrm{NaOH}$-treated and untreated pristine PVDF membranes and UV-curable resin-modified PVDF membrane

Ogata 1983; Kise et al. 1989), the PVDF membrane may be eroded into another substance after $\mathrm{NaOH}$ treatment (30 days), whose structure was

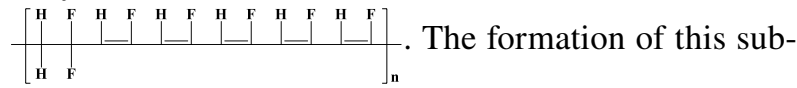

stance was resulted from severe dehydrofluorination of PVDF, which was consistent with the following XPS characterization results below. After severe dehydrofluorination, countless conjugated $\mathrm{C}=\mathrm{C}$ bonds generated in the PVDF main chain, resulting in difficulty in rotation in the polymer segment and increased rigidity. An asymmetric structure was formed in the polymer segment, so that the intermolecular interaction force and melting enthalpy become larger. The high melting enthalpy of peak $2 \#(291.6 \mathrm{~J} / \mathrm{g})$ was in line with our expected results.

When the UV-curable resin-modified PVDF membrane was treated with $\mathrm{NaOH}(\mathrm{pH}=13)$ for one month, two melting peaks appeared at $168.23^{\circ} \mathrm{C}$ and $153.40^{\circ} \mathrm{C}$, respectively. The peak $1 \#\left(168.23^{\circ} \mathrm{C}\right)$ of the UV-curable resin-modified membrane treated with $\mathrm{NaOH}$ is not much different from the melting peak of the UV-curable resin-modified membrane. But the melting temperature of peak $2 \#\left(153.4^{\circ} \mathrm{C}\right)$ is $14.83{ }^{\circ} \mathrm{C}$ lower than that of peak $1 \#\left(168.23^{\circ} \mathrm{C}\right)$. According to the above speculation, the corresponding substance of peak 2\# should be similar to

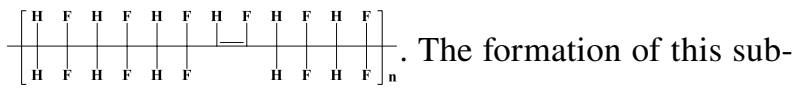
stance was contributed to mild dehydrofluorination of PVDF which formed isolated $\mathrm{C}=\mathrm{C}$ on the $\mathrm{PVDF}$ main chain. Thus, the $\mathrm{C}-\mathrm{C}$ bond besides the $\mathrm{C}=\mathrm{C}$ bond is easy to rotate and the flexibility of the polymer segment is better than that of the alkali-treated pristine membrane, while the intermolecular interaction force does not change much. Compared with the peak $2 \#(291.6 \mathrm{~J} / \mathrm{g})$ of the alkali-treated pristine membrane, 
the melting enthalpy value became smaller in line with our prediction. The DSC test results also verified our speculation. From the characterization results of DSC melting enthalpy, the alkali-resistant barrier effectively shielded the $\mathrm{NaOH}$ from attacking the PVDF membrane (Table 2).

\section{Contact angle analysis}

The largest change of the pristine membrane before and after the alkaline treatment in water contact angle can be observed in Fig. 8. Since the three lone pairs of fluorine on the carbon-fluorine bond are tightly fixed due to the high electronegativity of the atom, it is difficult to act as a hydrogen bond acceptor to interact with water molecules (O'Hagan 2008; Hunter 2010). This leads to the hydrophobic nature of the PVDF membrane material, and the contact angle of the pristine PVDF membrane is the result of the interaction between the additive hydrophilic nano-silica and the hydrophobic nature of PVDF itself. After the alkali treatment, the removal of the hydrogen fluoride unit from PVDF leads to a decrease in the carbon-fluorine bond, and the content of hydrophilic silica in the pristine membrane is almost unchanged (XPS characterization proves this), resulting in a decrease in the contact angle of pristine membrane (Zheng 2010). After PVDF was modified by UV-curing resin, the contact angle of the membrane decreased for the $\mathrm{C}=\mathrm{O}$ and $\mathrm{C}-\mathrm{Cl}$ group from resin could easily formed hydrogen bonds with water molecules. The contact angle of the membrane is slightly reduced after alkali treatment. The reason was that the SDS wrapped in the resin was the alkali-resistant barrier of PVDF, which can effectively shield the nucleophile $(\mathrm{NaOH})$ from attacking PVDF, thereby inhibiting the dehydrofluorination process; hydrogen fluoride is removed in a small amount from the polymer molecular chain, which leads to the lower drop in contact angle.

Table 2 The effect of $\mathrm{NaOH}$ treatment on melting temperature and melting enthalpy

\begin{tabular}{llllll}
\hline Specimen & \multicolumn{2}{l}{$\begin{array}{l}\text { Melting tempera- } \\
\text { ture }\left({ }^{\circ} \mathrm{C}\right)\end{array}$} & & $\begin{array}{l}\text { Melting enthalpy } \\
(\mathrm{J} / \mathrm{g})\end{array}$ \\
\cline { 2 - 3 } \cline { 5 - 6 } & Peak 1\# & Peak 2\# & Peak 1\# & Peak 2\# \\
\hline $\begin{array}{l}\text { M-Pristine } \\
\begin{array}{l}\text { M-Pristine after alkali treat- } \\
\text { ment }\end{array}\end{array}$ & 170.76 & - & 57.64 & - \\
$\begin{array}{l}\text { M-UV curing } \\
\begin{array}{l}\text { M-UV curing after alkali } \\
\text { treatment }\end{array}\end{array}$ & 170.30 & 110.52 & & 26.90 & 291.6 \\
\hline
\end{tabular}

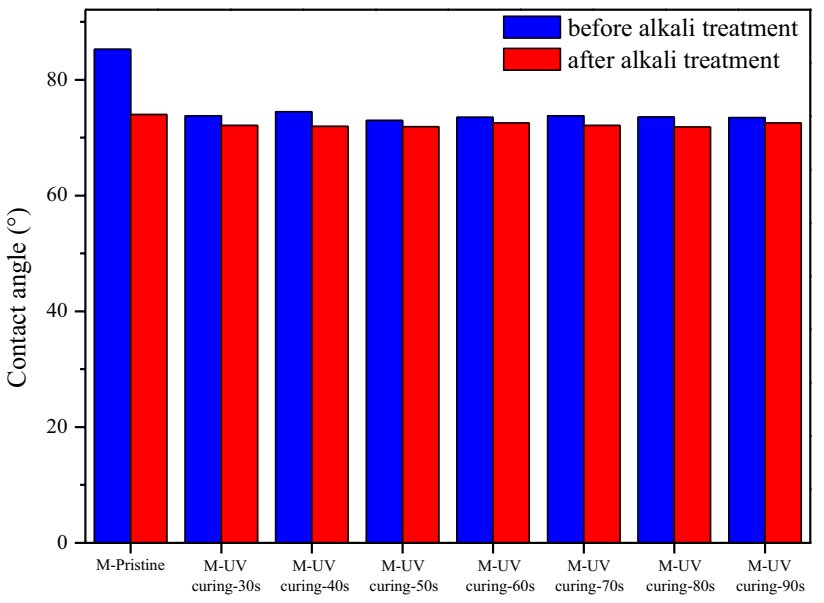

Fig. 8 The effect of alkali treatment on membrane contact angle

\section{Pore size distribution analyses}

As can be seen from Fig. 9a, the pore size of the membrane was reduced when PVDF was modified by ultraviolet curing resin. With the extension of curing time, the pore size of the membrane shows a tendency to become smaller, which was caused by the longer time polymerization of polyester acrylate and TPGDA, the resin formed wrapped the fingerlike and the sponge-like cross-sectional structure further, less unpolymerized polyester acrylate and TPGDA were removed when the membrane was soaked in ethanol. In short, the extension of the curing time caused the resin to further occupy the cross-sectional pores of the membrane and decrease of pore size.

As can be seen from Fig. 9b, both the pristine PVDF membrane and the UV-cured modified PVDF membrane, the pore size basically remained intact after the alkali treatment. As far as the pristine membrane is concerned, although the alkali treatment led to a decrease in the tensile strength of the membrane and dehydrofluorination, it did not change the physical structure of the PVDF membrane (Hashim et al. 2011a). For the UV-curable resin-modified PVDF membrane, the resin cured on the surface and cross section of the membrane has a strong alkali resistance (Cao and Zheng 2005), which is high resistant to the chemical attack of $\mathrm{NaOH}$. It was proved that the cured resin and PVDF membrane did not peel off (if not, the pore size of UV-curable resin-modified PVDF membrane will increase after alkali treatment). The above SEM characterization results also prove this.

\section{Pure water flux}

It can be seen from Fig. 10 that the pristine PVDF membrane has the largest pure water flux. After the ultraviolet curing 

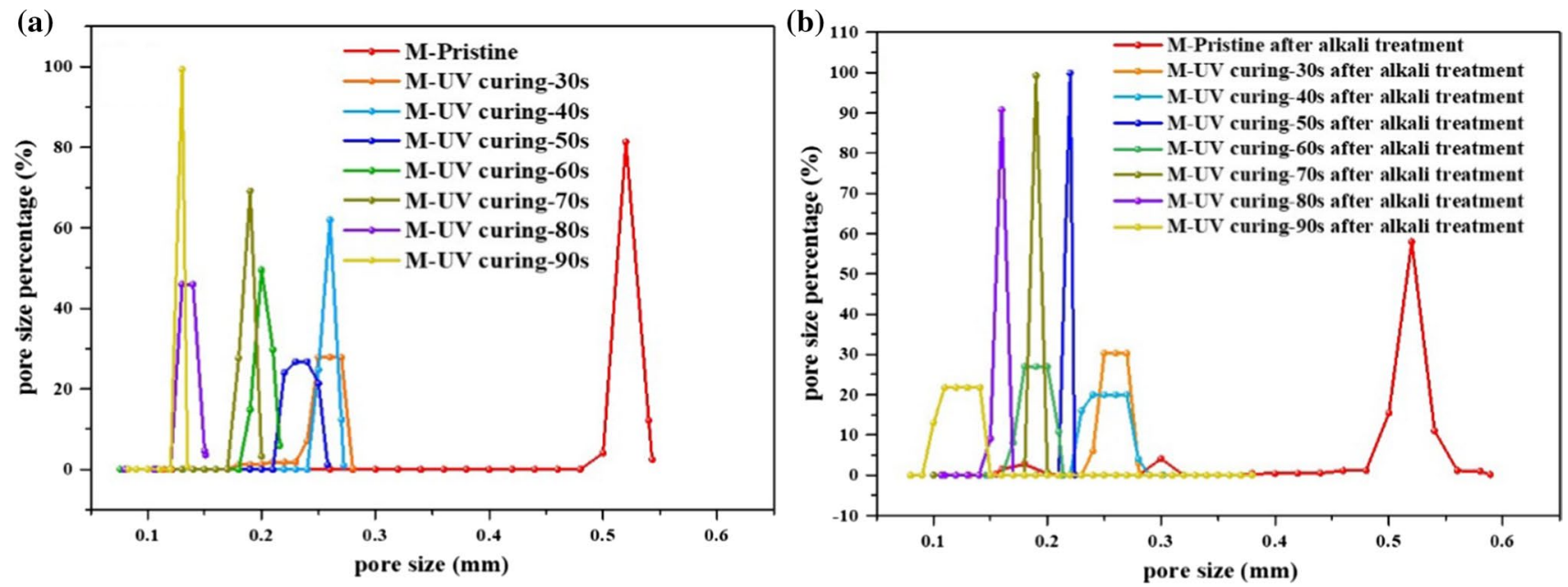

Fig. 9 a The effect of curing time on pore size before alkali treatment. b The effect of alkali treatment on membrane pore size distribution

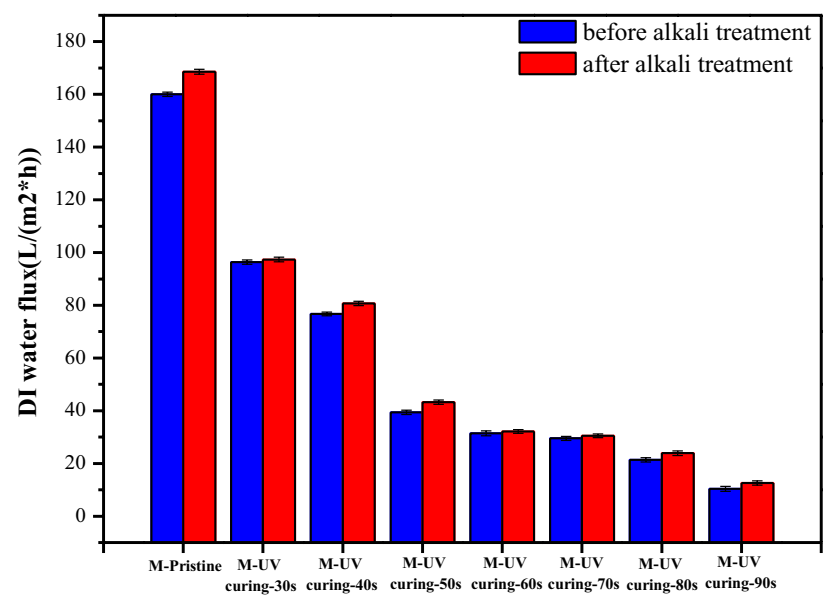

Fig. 10 The influence of alkali treatment on membrane pure water flux

resin-modified the PVDF membrane, the flux of the PVDF membrane decreased. With the extension of the curing time, the flux showed a tendency to decrease. The cause of the reduction has been analyzed in the characterization of pore size distribution characterization.

After the alkali treatment, the flux of the pristine membrane increased more than that of the UV-cured modified membrane. The reason is that although the size of the pristine membrane did not change after the alkali treatment, the PVDF membrane underwent severe dehydrofluorination. Therefore, the hydrophilic permeability significantly improved (Luo 2020). The large increase in flux is also reasonable. In terms of UV-curable resin-modified membrane, first of all, the pore size did not change. Second, the membrane was only slightly dehydrofluorinated by $\mathrm{NaOH}$ $(\mathrm{pH}=13)$, and the hydrophilic permeability did not change much, so the flux increase was smaller than that of the pristine PVDF membrane.

\section{XPS}

The XPS results of the UV-cured PVDF membrane (30-90 s) treated by alkali treatment are basically the same. Therefore, we chose a PVDF membrane with a curing time of $40 \mathrm{~s}$ to represent all the alkali-treated UV-cured membranes.

It can be seen from Table 3 that the carbon atom content increased by $2.21 \%$, the oxygen atom increased by $0.91 \%$, and the fluorine atom content decreased by $3.12 \%$ after UV-curing modification. The reason is that the cured resin itself attached to the PVDF membrane contains carbon and oxygen atoms, resulting in an increase in the ratio of oxygen atoms to carbon atoms, and a relatively low content of fluorine atoms.

XPS results show that the $\mathrm{F} / \mathrm{C}$ ratio of the pristine PVDF membrane was reduced from 69.28 to $50 \%$ after alkali treatment, indicating that dehydrofluorination occurred and led to the degradation of the PVDF membrane. However, the F/C ratio of the UV-cured PVDF membranes was reduced from 61.26 to $58.66 \%$ after immersion in alkaline solutions; the change in the F/C ratio was relatively low for the UV-curable resin-modified PVDF membrane compared to pristine PVDF membrane. Thus, it can be concluded that the dehydrofluorination of the UV-curable resin-modified PVDF membranes was lower than that of pristine PVDF membrane. The reason is that the resin formed by TPEDA and polyester acrylate 
Table 3 The effect of $\mathrm{NaOH}$ treatment on element of PVDF membrane

\begin{tabular}{llcc}
\hline Specimen & BE $(\mathrm{eV})$ & Atomic conc. (\%) & Error (\%) \\
\hline M-Pristine-C & 283.10 & 55.80 & 0.42 \\
M-Pristine-O & 529.20 & 5.54 & 0.28 \\
M-Pristine-F & 684.80 & 38.66 & 0.38 \\
M- Pristine after alkali treatment-C & 283.10 & 62.98 & 0.42 \\
M- Pristine after alkali treatment-O & 529.20 & 5.53 & 0.30 \\
M- Pristine after alkali treatment-F & 684.80 & 31.49 & 0.37 \\
M- UV curing -C & 283.10 & 58.01 & 0.42 \\
M- UV curing -O & 529.20 & 6.45 & 0.29 \\
M- UV curing -F & 684.80 & 35.54 & 0.37 \\
M- UV curing after alkali treatment-C & 283.10 & 58.96 & 0.43 \\
M- UV curing after alkali treatment-O & 529.20 & 6.46 & 0.30 \\
M- UV curing after alkali treatment-F & 684.80 & 34.59 & 0.37 \\
\hline
\end{tabular}

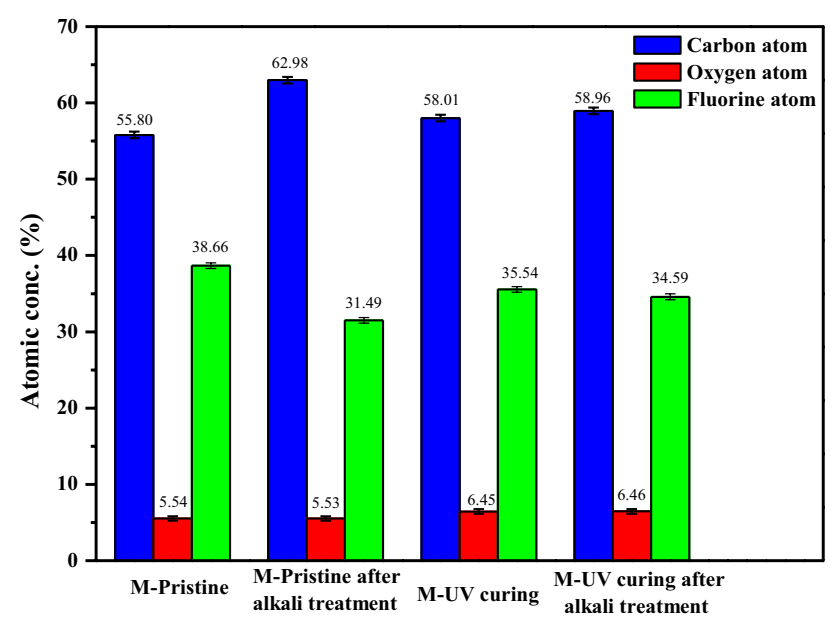

Fig. 11 The effect of $\mathrm{NaOH}$ treatment on element of PVDF membrane

firmly immobilize the SDS on the surface of the membrane, thereby resisting the chemical attack of the nucleophile NAOH and inhibiting dehydrofluorination. In addition, the excellent alkali corrosion resistance and water proof of the resin attached to the membrane surface will hinder the loss of SDS and give the membrane long-lasting alkali corrosion resistance (Fig. 11).

Regardless if it is pristine PVDF membrane or UV-curable resin-modified PVDF membrane, the content of oxygen atoms almost did not change before and after the alkaline solution treatment. As far as the pristine membrane is concerned, it shows that the influence of the alkaline solution on the inorganic nano-silica can be almost ignored. In case of UV-curable resin-modified PVDF membrane, this indicates that the resin attached to the PVDF membrane did not degrade, which demonstrates that the resin has excellent alkali resistance (Cao and Zheng 2005).

\section{Conclusions}

In this work, a novel alkali-resistant PVDF membrane via immobilization of sodium lauryl sulfate (SDS) was fabricated. The alkali-resistant performance of pristine PVDF and the UV-curable resin-modified PVDF membrane was evaluated by immersing in $\mathrm{NaOH}(\mathrm{pH}=13)$ solution for 30 days, respectively. The results reveal that the reduction in tensile strength of the UV-curable resin-modified PVDF membrane is much smaller than that of the pristine PVDF membrane under the same alkaline conditions. The UV-curable resinmodified PVDF membrane inhibits the chemical reaction of dehydrofluorination effectively compared with the pristine PVDF membrane, which has been certified by whiteness, DSC and XPS characterization. The successful curing of polyester acrylate and TPGDA on PVDF membrane was further confirmed by FTIR spectroscopy and SEM results. The extension of the curing time will cause the aperture of UV-curable resin-modified PVDF membrane to show a decreasing trend. The degradation degree of the UV-curable resin-modified PVDF membrane was much lower than that of the pristine PVDF membrane. The overall results predict the promising potential of anionic SDS and resin formed for alkali-resistant modification of PVDF membranes. This work paves the way to designing alkali-resistant membranes.

Supplementary Information The online version contains supplementary material available at https://doi.org/10.1007/s13201-021-01377-6.

Acknowledgements The authors would like to express their appreciation for the financial support of the Priority Academic Program Development of Jiangsu Higher Education Institution (PAPD), The National Natural Science Foundation of China (22078146), The Natural Science Foundation of Jiangsu Province (BK20200091), the Materials-Oriented Chemical Engineering State Key Laboratory Program (KL19-04), the Six Talent Peaks of Jiangsu provincial (JNHB-036), and the Deputyship for Research and Innovation, Ministry of Education in Saudi Arabia for funding this research work through the project number (632). 
Funding This study was funded by National Natural Science Foundation of China (Grant No. 22078146), State Key Laboratory of Materials-Oriented Chemical Engineering (Grant No. KL19-04), Deputyship for Research and Innovation, Ministry of Education in Saudi Arabia (Grant No. 632), Natural Science Foundation of Jiangsu Province (Grant No. BK20200091), Six Talent Peaks Project in Jiangsu Province (CN) (Grant No. JNHB-036), and Priority Academic Program Development of Jiangsu Higher Education Institutions (CN) (Grant No. PAPD).

\section{Compliance with ethical standards}

Conflict of interest There is no conflict of interest to declare.

Open Access This article is licensed under a Creative Commons Attribution 4.0 International License, which permits use, sharing, adaptation, distribution and reproduction in any medium or format, as long as you give appropriate credit to the original author(s) and the source, provide a link to the Creative Commons licence, and indicate if changes were made. The images or other third party material in this article are included in the article's Creative Commons licence, unless indicated otherwise in a credit line to the material. If material is not included in the article's Creative Commons licence and your intended use is not permitted by statutory regulation or exceeds the permitted use, you will need to obtain permission directly from the copyright holder. To view a copy of this licence, visit http://creativecommons.org/licenses/by/4.0/.

\section{References}

Antón E et al (2015) Ageing of polyethersulfone ultrafiltration membranes under long-term exposures to alkaline and acidic cleaning solutions. Chem Eng Sci 134:178-195

Boccaccio $T$ et al (2002) Characterization of PVDF membranes by vibrational spectroscopy. J Membr Sci 210(2):315-329

Cao Y, Zheng S (2005) Development of polyester acrylate luminous paint and its properties. J Mater Prot 38(12):62-64, 82

Cui $Z$ et al (2015) Crystalline polymorphism in poly(vinylidenefluoride) membranes. Prog Polym Sci 51:94-126

Ghasem N, Al-Marzouqi M, Duidar A (2012) Effect of PVDF concentration on the morphology and performance of hollow fiber membrane employed as gas-liquid membrane contactor for $\mathrm{CO} 2$ absorption. Sep Purif Technol 98:174-185

Hakeim OA, Abdelghaffar F, Haroun AA (2020) UV-curable hyperbranched polyester acrylate encapsulation of phthalocyanine pigments for high performance synthetic fabrics printing. Dyes Pigments 177:108307. https://doi.org/10.1016/j.dyepig.2020.108307

Han W (2014) Preparation of UV-curable hyperbranched polyester acrylate/SiO_2 hybrid materials. Polym Mater Sci Eng 30(12):160-164, 171

Hashim NA, Liu F, Li K (2009) A simplified method for preparation of hydrophilic PVDF membranes from an amphiphilic graft copolymer. J Membr Sci 345(1-2):134-141

Hashim NA, Liu Y, Li K (2011a) Stability of PVDF hollow fibre membranes in sodium hydroxide aqueous solution. Chem Eng Sci 66(8):1565-1575

Hashim NA, Liu Y, Li K (2011b) Preparation of PVDF hollow fiber membranes using $\mathrm{SiO} 2$ particles: the effect of acid and alkali treatment on the membrane performances. Ind Eng Chem Res 50(5):3035-3040

Hoa SV, Ouellette P (1983) Stress corrosion cracking of poly(vinylidene fluoride) in sodium hydroxide. Polym Eng Sci 23(4):202-205
Hunter L (2010) The C-F bond as a conformational tool in organic and biological chemistry. Beilstein J Organ Chem 6:38. https://doi. org/10.3762/bjoc.6.38

Jang Y-S et al (2017) Effects of sodium hydroxide cleaning on polyvinylidene fluoride fouled with humic water. Membr Water Treat $8(2): 149-160$

Kise H, Ogata H (1983) Phase transfer catalysis in dehydrofluorination of poly(vinylidene fluoride) by aqueous sodium hydroxide solutions. J Polym Sci Part A-1 Polym Chem 21(12):3443-3451

Kise H, Ogata H, Nakata M (1989) Chemical dehydrofluorination and electrical conductivity of poly(vinylidene fluoride) films. Macromol Mater Eng 168(1):205-216

Komaki Y, Otsu H (1981) Observation of nuclear track development in polyvinylidene fluoride with several etchants. J Electron Microsc 30(4):292-297

Kuhn KJ et al (1987) Structural and quantitative analysis of surface modified poly(vinylidene fluoride) films using ATR FT-IR spectroscopy. Appl Spectrosc 41(5):843-847

$\mathrm{Li} \mathrm{H}$ et al (2008) Effects and model of alkaline waste activated sludge treatment. Biores Technol 99(11):5140-5144

Liu Q-F, Lee C-H, Kim H (2010) Performance evaluation of alkaline treated poly(vinylidene fluoride) membranes. Sep Sci Technol 45(9):1209-1215

Liu F et al (2011) Progress in the production and modification of PVDF membranes. J Membr Sci 375(1-2):1-27

Liu J et al (2017) Effect of different chemical cleaning agents on the ageing of PVDF ultrafiltration membrane. J Harbin Inst Technol 49(8):25-30

Lovinger AJ (1983) Ferroelectric polymers. Science 220(4602):1115-1121

Luo $S$ et al (2020) Influence of alkali treatment on the structure and dielectric properties of polyvinylidene fluoride. Polym Mater Sci Eng 36(5):42-48

Mansourizadeh A, Ismail AF, Matsuura T (2010) Effect of operating conditions on the physical and chemical $\mathrm{CO} 2$ absorption through the PVDF hollow fiber membrane contactor. J Membr Sci 353(1-2):192-200

O'Hagan D (2008) Understanding organofluorine chemistry. An introduction to the C-F bond. Chem Soc Rev 37(2):308-319

Rabuni MF et al (2013) Effects of alkaline environments at mild conditions on the stability of PVDF membrane: an experimental study. Ind Eng Chem Res 52(45):15874-15882

Ross GJ et al (2000) Surface modification of poly(vinylidene fluoride) by alkaline treatment: 1 . The degradation mechanism. Polymer 41(5):1685-1696

Shinohara H (1979) Fluorination of polyhydrofluoroethylenes-2. Formation of perfluoroalkyl carboxylic acids on the surface region of poly(vinylidene fluoride) film by oxyfluorination, fluorination, and hydrolysis. J Polym Sci Polym Chem Ed 17(5):1543-1556

Srisurichan S, Jiraratananon R, Fane AG (2005) Humic acid fouling in the membrane distillation process. Desalination 174(1):63-72

Vigo F et al (1984) Mechanical, chemical and bacterial resistance of modified polyvinylidene fluoride membranes suitable for ultrafiltration of oily emulsions. J Membr Sci 21(3):295-306

Wang Z et al (2014) Membrane cleaning in membrane bioreactors: a review. J Membr Sci 468:276-307

Wegener M, Küunstler W, Gerhard-Multhaupt R (2006) Poling behavior and optical absorption of partially dehydrofluorinated and uniaxially stretched polyvinylidene fluoride. Ferroelectrics 336:3-8

Wu Q, Zhang X, Cao G (2018) Impacts of sodium hydroxide and sodium hypochlorite aging on polyvinylidene fluoride membranes fabricated with different methods. J Environ Sci 67:294-308

Yeow ML, Liu YT, Li K (2003) Isothermal phase diagrams and phaseinversion behavior of poly(vinylidene fluoride)/solvents/additives/ water systems. J Appl Polym Sci 90(8):2150-2155

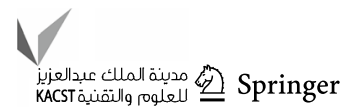


Yu H et al (2013) Disintegration and acidification of MBR sludge under alkaline conditions. Chem Eng J 231:206-213

Zhang S et al (2006) ESR and vibrational spectroscopy study on poly(vinylidene fluoride) membranes with alkaline treatment. J Power Sources 153(2):234-238

Zheng Z et al (2010) Superhydrophobic poly(vinylidene fluoride) film fabricated by alkali treatment enhancing chemical bath deposition. Appl Surf Sci 256(7):2061-2065
Zuo D-Y et al (2010) A study on submerged rotating MBR for wastewater treatment and membrane cleaning. Korean J Chem Eng 27(3):881-885

Publisher's Note Springer Nature remains neutral with regard to jurisdictional claims in published maps and institutional affiliations. 